\title{
Gliomatosis peritonei with bilateral ovarian teratomas: A report of two cases
}

\author{
JIAWEI WANG ${ }^{1}$, JINGJING XU ${ }^{1}$, MINMING ZHANG ${ }^{1}$ and BAIZHOU LI $^{2}$ \\ Departments of ${ }^{1}$ Radiology and ${ }^{2}$ Pathology, The Second Affiliated Hospital, Zhejiang University School of Medicine, \\ Hangzhou, Zhejiang 310009, P.R. China
}

Received March 4, 2015; Accepted June 17, 2016

DOI: $10.3892 / \mathrm{ol} .2016 .4866$

\begin{abstract}
Gliomatosis peritonei (GP) is characterized by the presence of benign, mature neuroglial implants throughout the peritoneum and is typically accompanied by mature or immature ovarian teratomas. GP is a condition that has only been described relatively recently, with $\sim 100$ cases reported in the English literature. The majority of reported cases have focused on the pathology and clinical treatment of the disease; radiological findings are distinct, but the discussion of this is scarce in the literature. The current study presents two cases of GP with bilateral ovarian teratomas and provides a review of the relevant literature, with particular emphasis on the radiological differential diagnosis. The present study reinforces previously reported observations from imaging analysis and suggests that radiological investigation alone cannot sufficiently aid the differentiation of benign glial deposits from diffuse peritoneal malignant seeding. However, radiologists should be familiar with this rare condition in order to provide an accurate diagnosis, particularly in ovarian tumor staging, which may markedly impact the administered treatment. It is recommended that doctors undertake long-term follow-ups in patients presenting with GP due to the potential for malignant transformation.
\end{abstract}

\section{Introduction}

Gliomatosis peritonei (GP) is a rare condition that is typically associated with immature and, less commonly, mature teratomas and ventriculoperitoneal shunting. GP occurs commonly in young girls and, in rare cases, can be found in males. GP is characterized as a myriad of peritoneal nodular or miliary implants composed of benign, mature glia (1). As GP has an extremely low incidence rate, there is no widely

Correspondence to: Dr Jingjing Xu, Department of Radiology, The Second Affiliated Hospital, Zhejiang University School of Medicine, 88 Jiefang Road, Hangzhou, Zhejiang 310009, P.R. China E-mail:mcdxjj@163.com

Key words: gliomatosis peritonei, ovarian teratoma, Pseudo-Meigs' syndrome, peritoneal dissemination, computed tomography accepted guidance as to the method by which these patients should be treated. Approximately 100 cases of GP have been described in the literature. Since the disease was first described by Neuhaüser in 1906 (2), it has been well-documented that GP, when benign, does not adversely affect patient prognosis. Glial implants associated with the disease may remain stable for long periods of time, or may completely disappear. The repeated measurement of tumor markers and imaging are usually undertaken throughout follow-up. To the best of our knowledge, only a few cases have been reported that describe the findings of a radiological analysis (3-5). The current study presents two cases of GP with bilateral ovarian teratomas, describing the diagnosis, associated radiological observations and complete clinical course of each patient.

\section{Case report}

Case 1. A 19-year-old woman was referred to The Second Affiliated Hospital, Zhejiang University School of Medicine (Hangzhou, China) on January 16, 2011, presenting with a 15-day history of lower abdominal distension. During a physical examination of the pelvis and abdomen, a large firm mass was identified and was palpable extending up to $20 \mathrm{~cm}$ to the xiphoid process. Analysis of serum tumor markers demonstrated an increase in carcinoembryonic antigen (13.4 ng/ml; normal range, $<5 \mathrm{ng} / \mathrm{ml}$ ), cancer antigen (CA)125 (241.7 units/ml; normal range, $<35$ units $/ \mathrm{ml}$ ) and CA19-9 $(2,215.6$ units $/ \mathrm{ml}$; normal range, $<37$ units $/ \mathrm{ml})$, whilst the $\alpha$-fetoprotein level was normal $(1.2 \mathrm{ng} / \mathrm{ml}$; normal range, $<20 \mathrm{ng} / \mathrm{ml})$.

Contrast-enhanced computed tomography (CT) revealed two cystic, solid masses in the pelvis and lower abdomen, each containing dispersed regions of calcified and fatty components. The two masses demonstrated similarities with bilateral ovarian teratomas. Additionally, multiple nodules throughout the peritoneum were associated with omental caking, and ascites was also detected (Fig. 1A-C). Based on the results from the $\mathrm{CT}$ scan and the elevated serum tumor markers, it was suspected that the patient was presenting with malignant germ cell tumors, specifically immature teratomas, with lymphadenopathy.

A right salpingo-oophorectomy, left tumor resection and omentectomy were performed. The right ovarian mass was $30 \times 25 \times 20 \mathrm{~cm}$ in size, and consisted of a yellow-white solid 
A

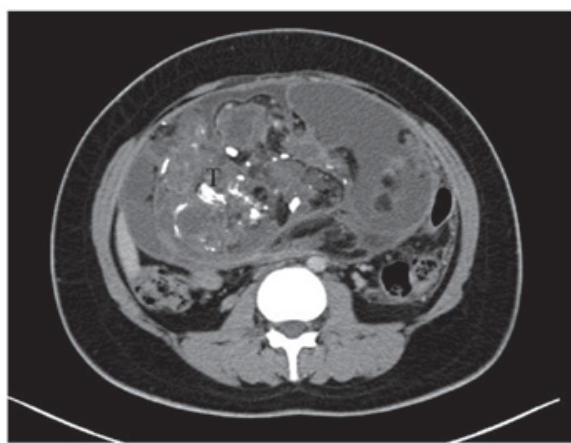

C

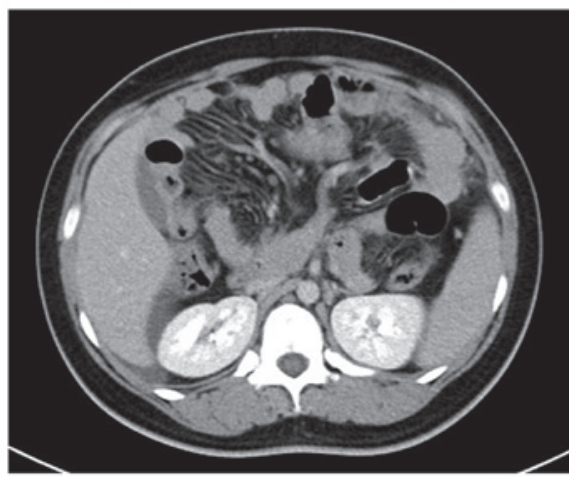

B

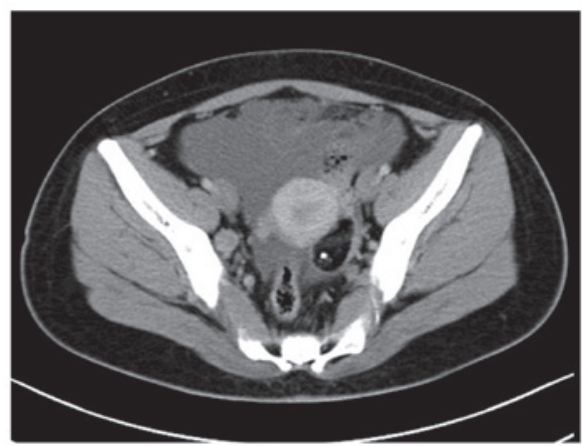

D

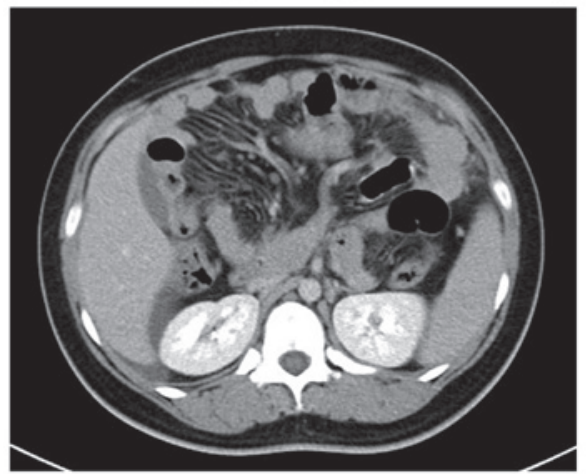

Figure 1. (A) Axial image of contrast-enhanced lower abdominal computed tomography demonstrated a mixed solid and cystic mass with scattered areas of fatty components and calcifications suggestive of an ovarian teratoma. Ascites was also observed. (B) Left ovarian teratoma and (C and D) multiple nodules throughout the peritoneum were exhibited. (A-C) Case 1. (D) Case 2.

A

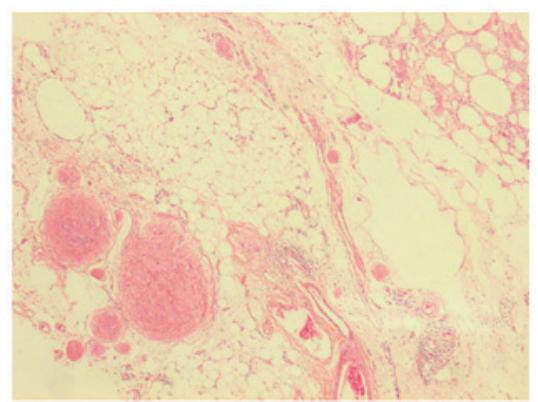

B

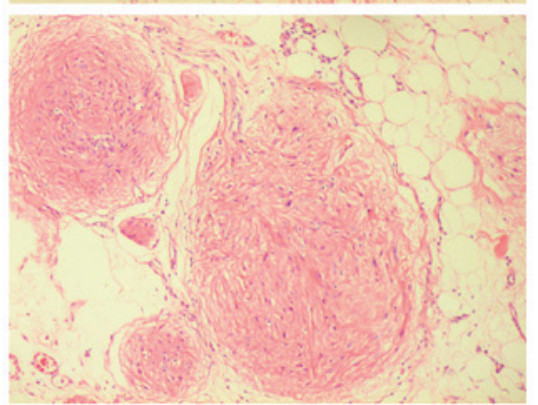

Figure 2. Case 1: (A) Multiple nodules demonstrating a variety of sizes in the greater omentum, with numerous lymphocytes and foam cells (staining, hematoxylin and eosin; magnification, x40). (B) Microscopic view of the omental implants exhibiting mature glial tissues, consistent with gliomatosis peritonei (staining, hematoxylin and eosin; magnification, x100).

component and cystic areas. Two cystic masses, measuring $1 \times 1$ and $2 \times 1 \mathrm{~cm}$, were observed in the left ovary. All tumors contained fatty components. Numerous miliary nodules were identified in the retroperitoneum and peritoneum, suggesting the presence of GP implants. The largest implant was detected in the greater omentum, measuring $1 \mathrm{~cm}$ in diameter, and had adhered to the right ovary. Following histological examination, the right ovarian mass was confirmed as an immature teratoma, and the left mass was confirmed as a mature teratoma. Mature teratoma was characterized by well-differentiated tissue, while immature teratoma contained immature tissue, typically the neuroectodermal structures. Extensive peritoneal implants were observed, with numerous lymphocytes and foam cells. The implants had no envelope, but were without cellular atypia or mitosis (Fig. 2).

Following surgery, a chemotherapy regimen (30 mg bleomycin on days $1-3,90 \mathrm{mg}$ etoposide on day 1 and $0.17 \mathrm{mg}$ cisplatin on day 1, for 6 cycles; interval time, 4 weeks) was administered. Currently, 4 years after the first surgery, the patient is alive and well, with a good prognosis and without recurrence or metastasis. A long-term follow-up is planned at an outpatient clinic.

Case 2. A 15-year-old woman was referred to The Second Affiliated Hospital, Zhejiang University School of Medicine on July 17, 2013, presenting with progressively increasing fullness of the abdomen and a significant increase in weight over the last 3 years. The patient was subsequently referred to the Department of Endocrinology for the appropriate treatment. Contrast-enhanced CT detected a tumor in the lower abdomen and pelvis, measuring 10x12x9 cm; a second tumor in the left ovary, with a diameter of $3 \mathrm{~cm}$, was also revealed on the CT scan (Fig. 1D). The patient was treated with a tumorectomy. During the surgery, bilateral tumors were found, and the larger tumor was located in the right ovary. The tumors were removed. Intraoperatively, tiny gray spots, measuring up to $0.5 \mathrm{~cm}$ in diameter, were removed from the greater omentum and peritoneum. During microscopic examination, the tumors 
appeared to consist of a teratomatous component, exhibiting similarities to skin, intestinal mucosa, cartilage, bones and neuroglial tissue. It was also observed that the peritoneal implants consisted of mature glial tissue that appeared to be well-demarcated, without mitosis or cellular atypia. Therefore, a diagnosis of bilateral mature teratoma and GP was confirmed. No chemotherapy was administered for case 2 . During a follow-up period of 25 months after surgery, no notable events were recorded and the patient has a good prognosis. A long-term follow-up is planned at an outpatient clinic.

\section{Discussion}

GP is a rare complication associated with ovarian teratomas, with only $\sim 100$ cases recorded to date. Typical symptoms of the disease are similar to those experienced in the present two cases, and affected patients may occasionally develop Pseudo-Meigs' syndrome associated with ascites and pleural effusions owing to ovarian teratoma (6). The pre-operative diagnosis of teratoma may be confirmed through the combination of imaging and clinical findings. However, small GP lesions may be easily overlooked or cause a delay in accurate diagnosis due to the rareness of the disease.

GP is characterized by a myriad of peritoneal nodular or miliary implants composed of mature glia (7). As mature glial cells are not aggressive, the implants may remain stable for long periods of time, exhibiting benign behavior. However, on rare occasions, GP may develop a secondary malignant glial tumor (8). The pathogenesis underlying the development of GP is not yet fully understood. These glial nodules are traditionally confined as peritoneal seedlings via capsular rupture from the ovarian teratoma, although genetic evidence has demonstrated that they may have a different genetic identity compared with ovarian tumors (9-11). In the majority of cases, a benign clinical course is expected for patients with GP, particularly when undergoing cisplatin-based chemotherapy (12). In the present two cases, following surgical resection, each patient demonstrated a positive prognosis within a 19-month follow-up period. However, doctors should conduct long-term follow-ups in patients presenting with GP due to the potential for malignant transformation.

When comparing the radiological data from the present cases with previously published cases, notable findings have been observed: i) Although the teratomas in the present cases were localized on each ovary (bilateral), the majority of cases within the literature reported unilateral teratomas, without a particular preference as to which ovary was affected; up until now, only one case of bilateral teratoma had been reported in the literature (13). ii) Although the radiological data regarding GP in the current literature is scarce, the radiological findings in the present two cases appear to be consistent with previous observations. The CT scans from the current study revealed multiple nodules throughout the peritoneum, with associated omental caking and ascites. The peritoneal implants were miliary, measured $0.3-1.2 \mathrm{~cm}$ in size, and were well-marginated and markedly enhanced $(4,5,14)$. The findings in the two present cases are similar to previous observations of peritoneal tuberculosis and peritoneal dissemination of malignant tumors (5). Therefore, with regard to the identification of ovarian masses, imaging analysis of peritoneal dissemination alone cannot aid the differentiation of benign implants from diffuse peritoneal malignant seeding. However, radiological analysis may provide important information regarding tumor staging and the requirement for surgery. Therefore, in patients with bilateral ovarian teratomas, GP should be considered as a possible diagnosis by radiologists and relative radiological investigations are warranted.

In summary, the current study reports two cases presenting with bilateral ovarian teratomas with GP. These cases suggest that: i) In patients with ovarian teratoma, a possible diagnosis of GP should be considered, particularly in individuals presenting with bilateral teratomas; ii) although radiology may provide biological and histopathological information regarding GP status, the radiological differential diagnosis of GP is challenging due to the rareness of the disease; and iii) the follow-up of patients with GP should not be discontinued following the initial diagnosis and treatment, even if symptoms are alleviated or disappear.

\section{References}

1. Nogales FF, Dulcey I and Preda O: Germ cell tumors of the ovary: An update. Arch Pathol Lab Med 138: 351-362, 2014.

2. Neuhaüser H: On teratoid tumors of the ovary. Arch Gynaek 79: 696-719, 1906 (In German).

3. Brammer HM III, Buck JL, Hayes WS, Sheth S and Tavassoli FA: From the archives of the AFIP. Malignant germ cell tumors of the ovary: Radiologic-pathologic correlation. Radiographics 10: 715-724, 1990.

4. Kishimoto K, Ito K, Furukawa M, Ogasawara N, Matsunaga N, Nawata $\mathrm{S}$, Ogata $\mathrm{H}$ and Kato $\mathrm{H}$ : Immature teratoma with gliomatosis peritonei associated with pregnancy. Abdom Imaging 27: 96-99, 2002

5. Okamoto D, Ishigami K, Yoshimitsu K, Irie H, Tajima T, Asayama Y, Hirakawa M, Ushijima Y, Nishihara Y, Amada S, et al: Gliomatosis peritonei associated with immature ovarian teratoma: A mimicker of peritoneal dissemination of malignant diseases. J Comput Assist Tomogr 31: 317-319, 2007.

6. Khan J, McClennan BL, Qureshi S, Martell M, Iyer A and Bokhari SJ: Meigs syndrome and gliomatosis peritonei: A case report and review of literature. Gynecol Oncol 98: 313-317, 2005.

7. Menéndez-Sánchez P, Villarejo-Campos P, Padilla-Valverde D, Murillo-Lázaro C and Martín-Fernández J: Gliomatosis peritonei: Recurrence, treatment and surveillance. Cir Cir 79: 256-259, 2011.

8. Dadmanesh F, Miller DM, Swenerton KD and Clement PB: Gliomatosis peritonei with malignant transformation. Mod Pathol 10: 597-601, 1997.

9. Ferguson AW, Katabuchi H, Ronnett BM and Cho KR: Glial implants in gliomatosis peritonei arise from normal tissue, not from the associated teratoma. Am J Pathol 159: 51-55, 2001.

10. Kwan MY, Kalle W,Lau GT and Chan JK: Is gliomatosis peritonei derived from the associated ovarian teratoma? Hum Pathol 35: 685-688, 2004

11. Best DH, Butz GM, Moller K, Coleman WB and Thomas DB: Molecular analysis of an immature ovarian teratoma with gliomatosis peritonei and recurrence suggests genetic independence of multiple tumors. Int J Oncol 25: 17-25, 2004.

12. Liang L, Zhang Y, Malpica A, Ramalingam P, Euscher ED, Fuller GN and Liu J: Gliomatosis peritonei: a Clinicopathologic and immunohistochemical study of 21 cases. Mod Pathol 28: 1613-1620, 2015.

13. Roscher AA, Weinstein EC and Powsner L: Giant teratomas with benign glial abdominal seeding, mimicking diffuse abdominal carcinomatosis. Int Surg 60: 461-465, 1975.

14. England RA, deSouza NM and Kaye SB: Gliomatosis peritonei: MRI appearances and its potential role in follow up. Br J Radiol 80: e101-e104, 2007. 